\title{
Design, Synthesis, Characterization, and Insecticidal Bio-efficacy Screening of Some New Pyridine Derivatives
}

Adel M. Kamal El-Dean, ${ }^{\mathrm{a}}$ Aly A. Abd-Ella, ${ }^{\mathrm{b}}$ Reda Hassanien, ${ }^{\mathrm{c}}$ Mohamed E. A. El-Sayed, ${ }^{\mathrm{d}}$ Shaban A. A. Abdel-Raheem ${ }^{\mathrm{d} *}$

${ }^{a}$ Chemistry Department, Faculty of Science, Assiut University, 71516 Assiut, Egypt

${ }^{\mathrm{b}}$ Plant Protection Department, Faculty of Agriculture, Assiut University, 71526 Assiut, Egypt

${ }^{c}$ Chemistry Department, Faculty of Science, New Valley University, El-Kharja, 72511, Egypt

${ }^{\mathrm{d}}$ Soil, Water, and Environment Research Institute, Agriculture Research Center, 12112 Giza, Egypt

* Corresponding author:

E-mail: mrshaban2000@gmail.com 


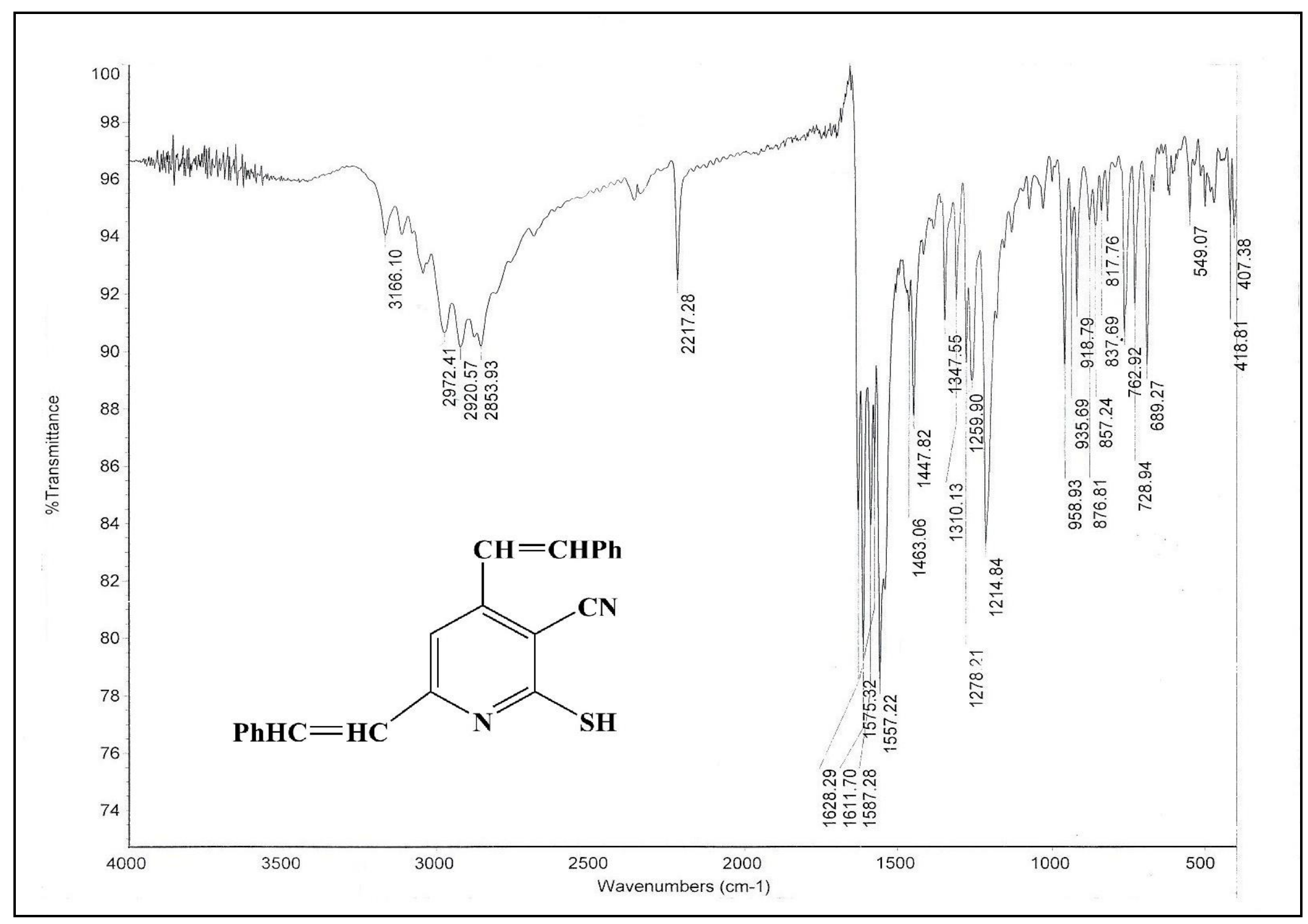

Figure S1: IR spectrum of 3-cyano-4,6-distyrylpyridine-2(1H)-thione (4). 


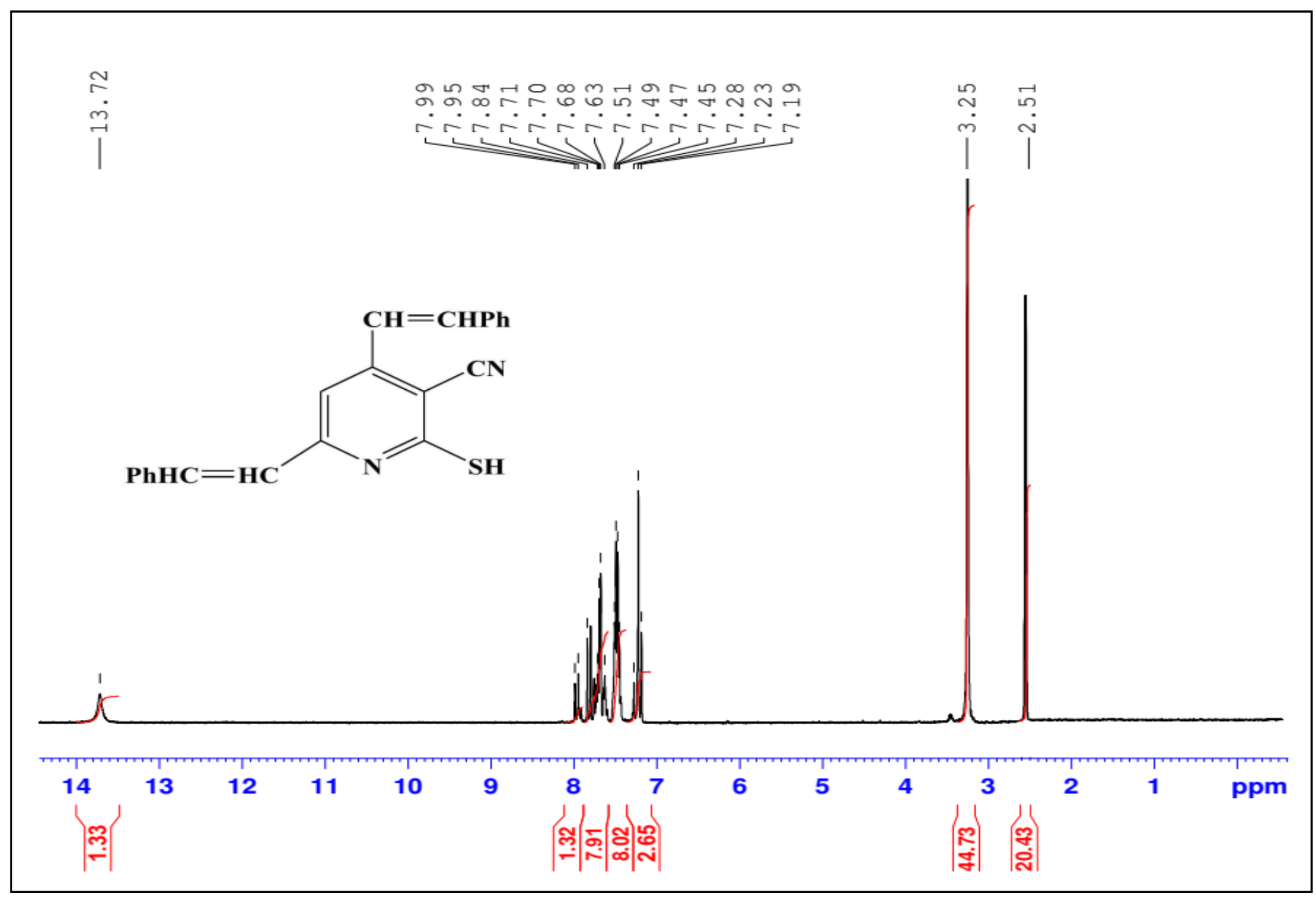

Figure S2: ${ }^{1} \mathrm{H}$ NMR spectrum of 3-cyano-4,6-distyrylpyridine-2(1H)-thione (4). In DMSO- $d_{6}$ 


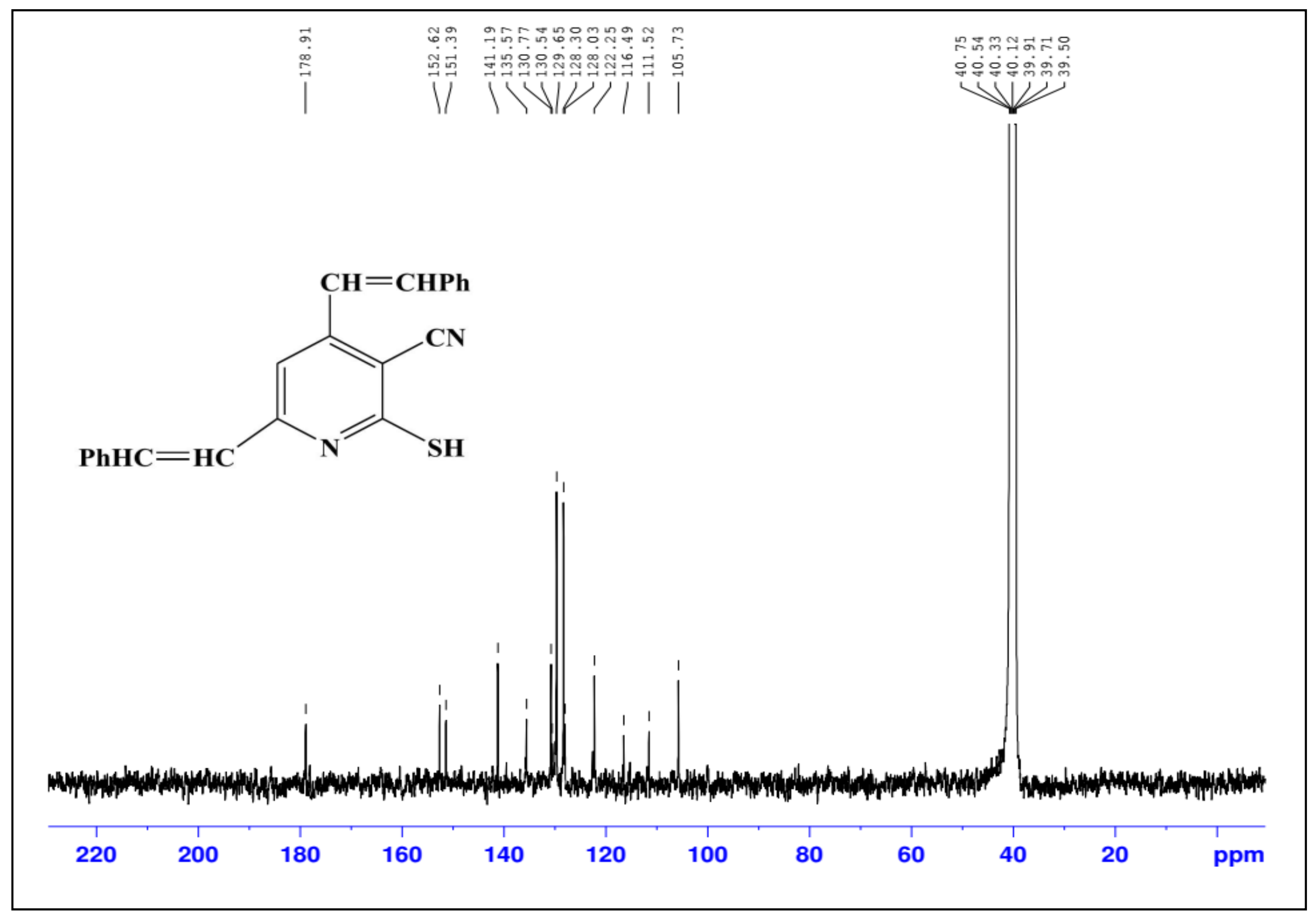

Figure S3: ${ }^{13} \mathrm{C}$ NMR spectrum of 3-cyano-4,6-distyrylpyridine-2(1H)-thione (4). In DMSO- $d_{6}$ 


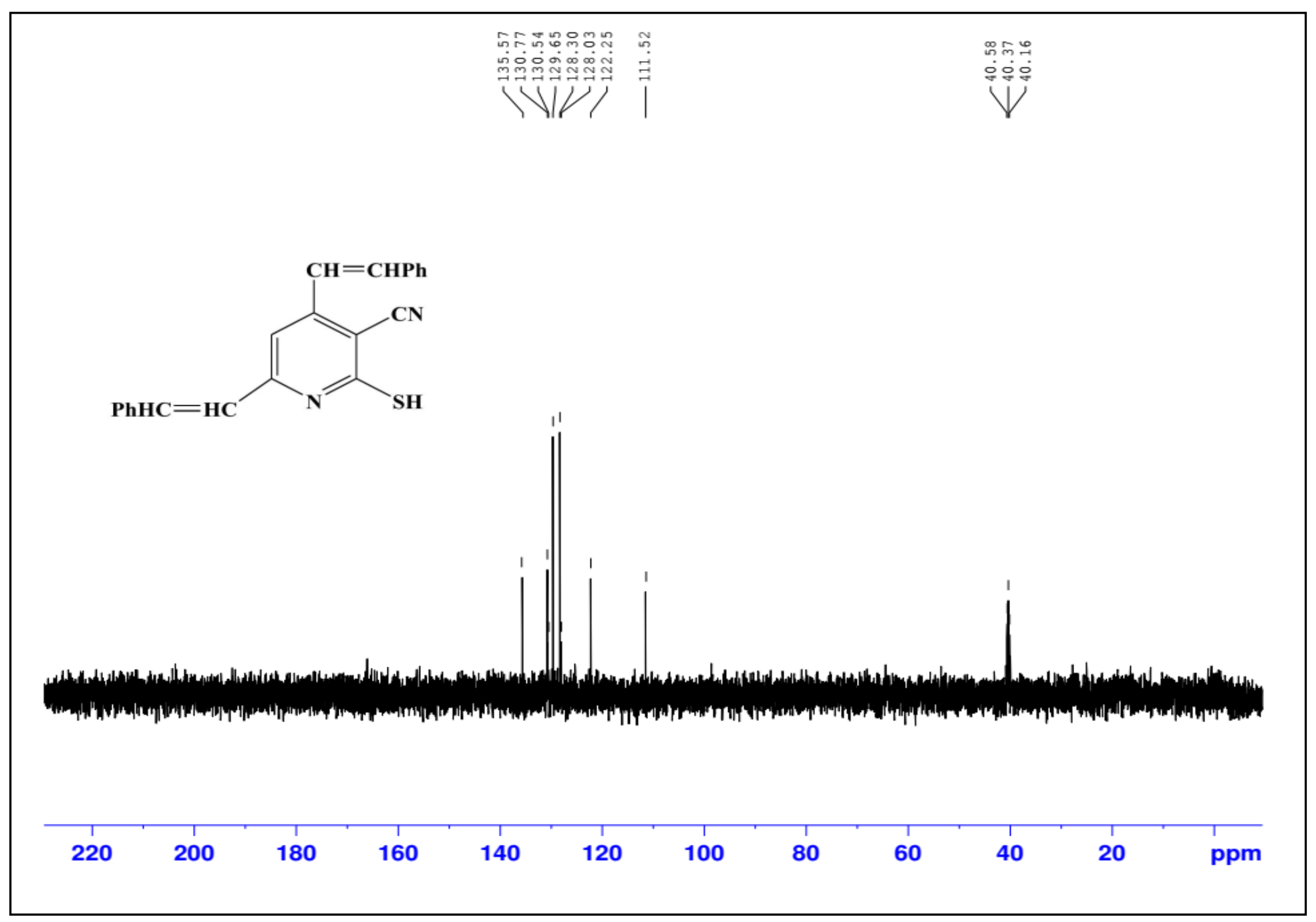

Figure S4: DEPT 135 spectrum of 3-cyano-4,6-distyrylpyridine-2(1H)-thione (4). In DMSO-d 6 


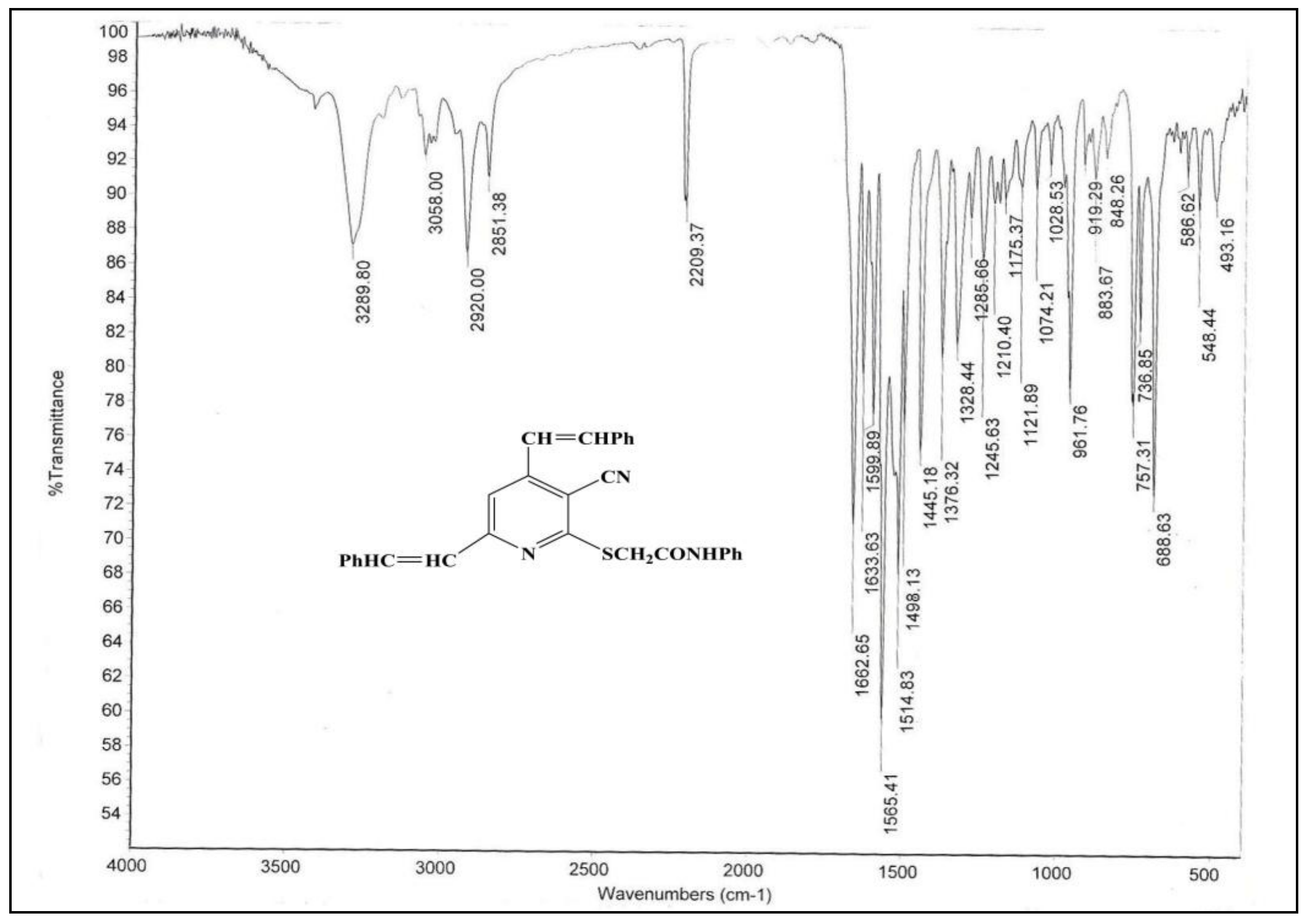

Figure S5: IR spectrum of 2-((3-cyano-4,6-distyrylpyridin-2-yl)thio)-N-phenylacetamide (5). 


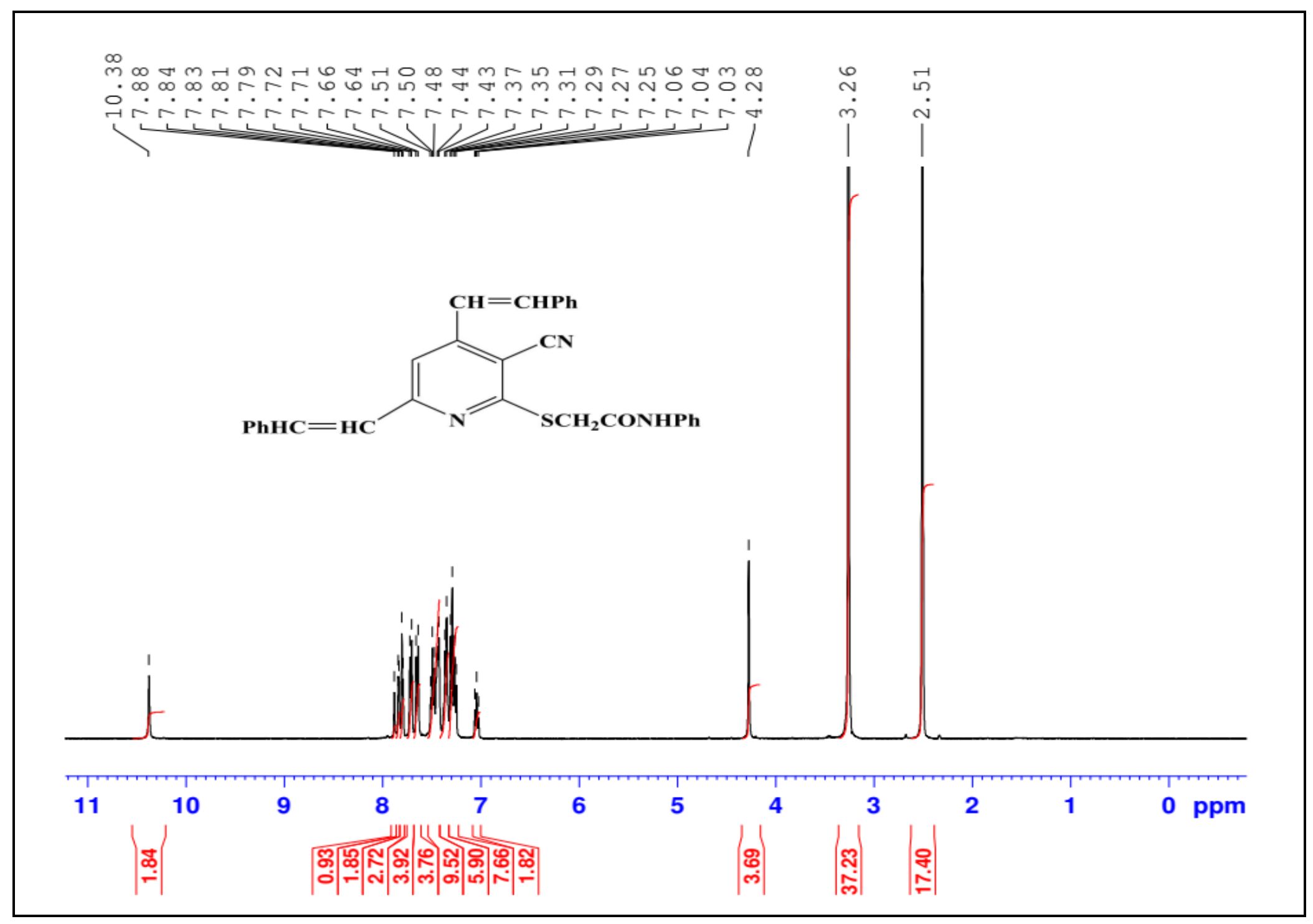

Figure S6: ${ }^{1} \mathrm{H}$ NMR spectrum of 2-((3-cyano-4,6-distyrylpyridin-2-yl)thio)-N-phenylacetamide (5). In DMSO-d 6 


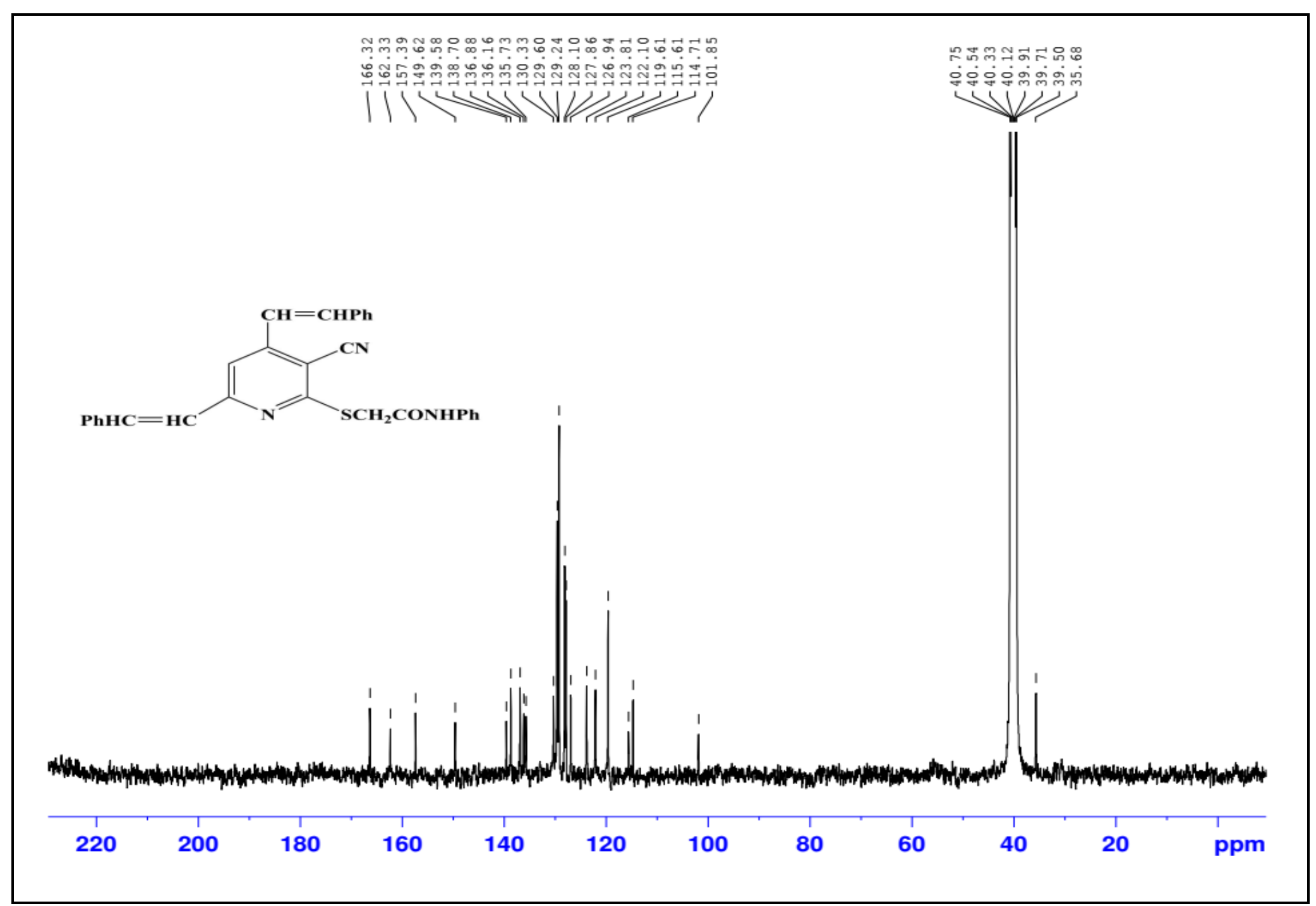

Figure S7: ${ }^{13} \mathrm{C}$ NMR spectrum of 2-((3-cyano-4,6-distyrylpyridin-2-yl)thio)-N-phenylacetamide (5). In DMSO- $d_{6}$ 


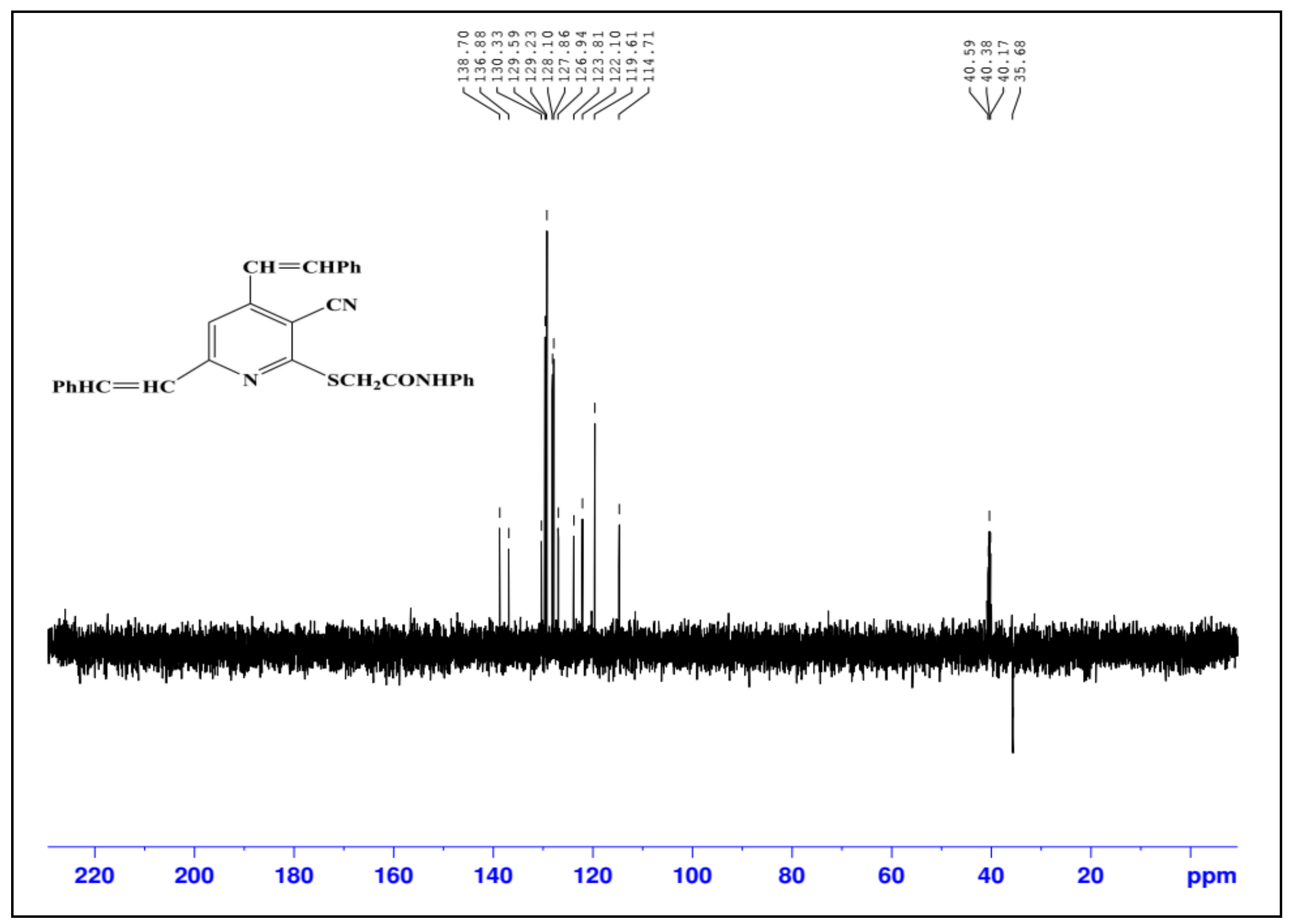

Figure S8: DEPT 135 spectrum of 2-((3-cyano-4,6-distyrylpyridin-2-yl)thio)-N-phenylacetamide (5). In DMSO-d 6 


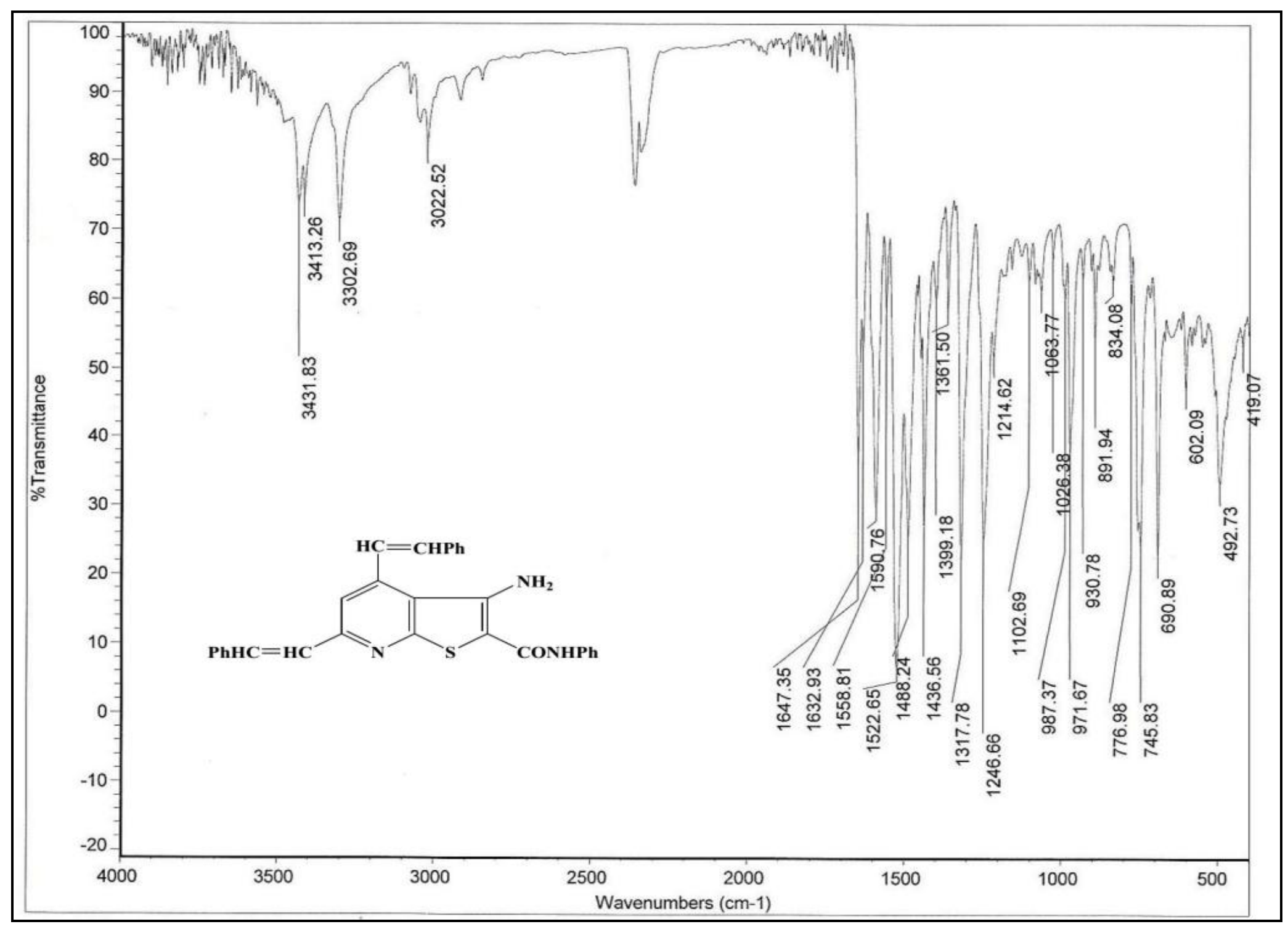

Figure S9: IR spectrum of 3-amino-N-phenyl-4,6-distyrylthieno[2,3-b]pyridine-2-carboxamide (6). 


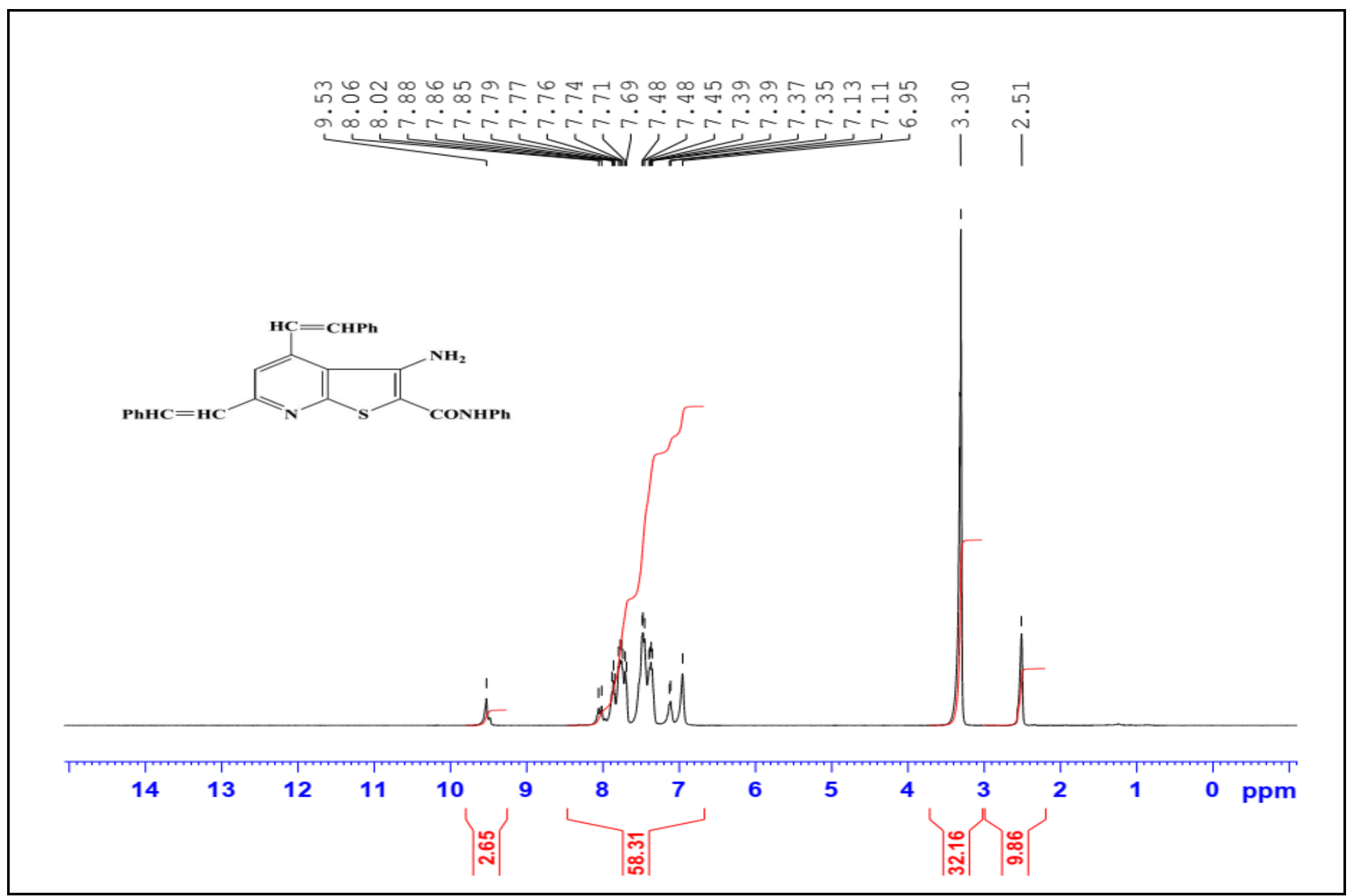

Figure S10: ${ }^{1} \mathrm{H}$ NMR spectrum of 3-amino-N-phenyl-4,6-distyrylthieno[2,3-b]pyridine-2-carboxamide (6). In DMSO-d 6 


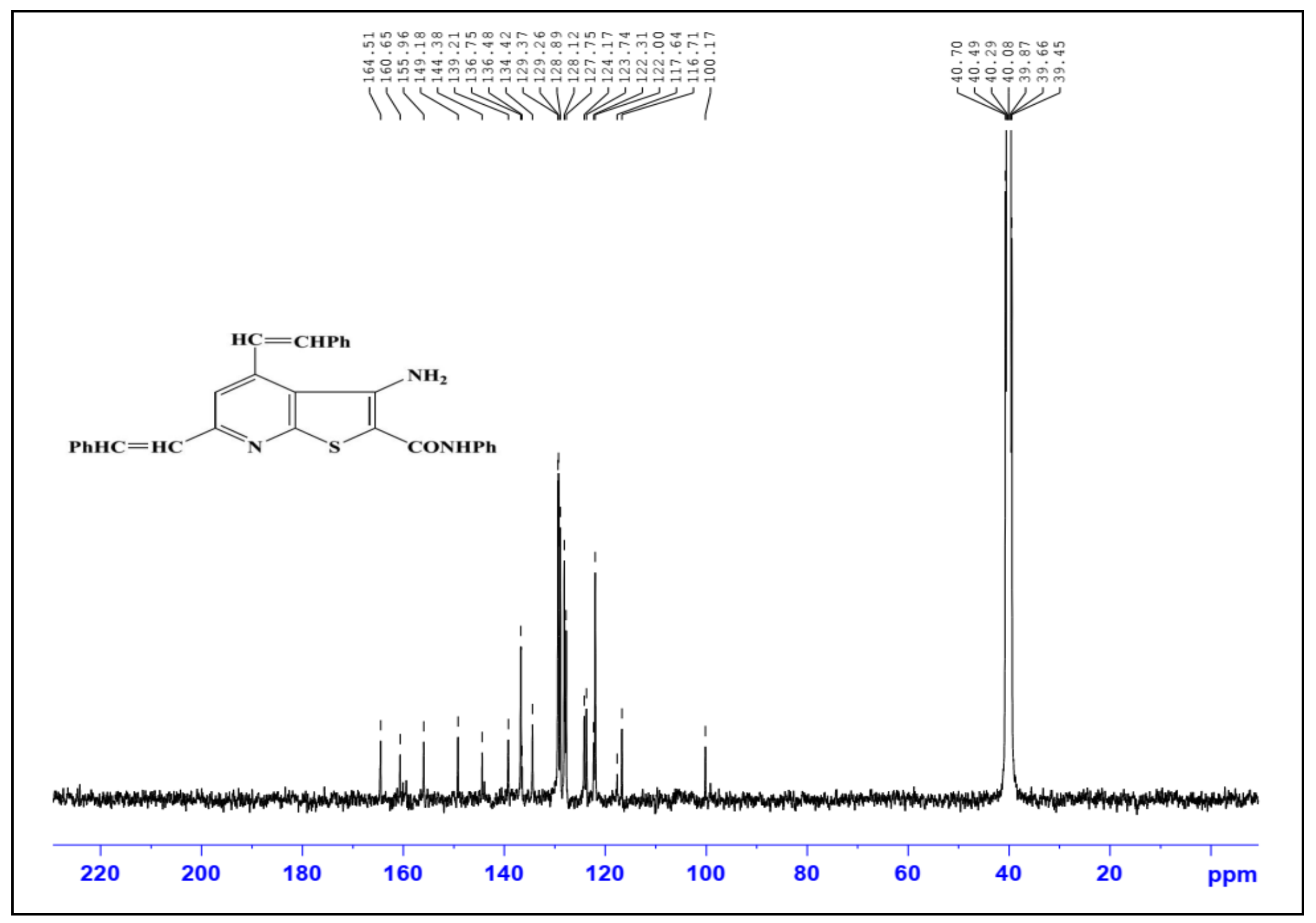

Figure S11: ${ }^{13} \mathrm{C}$ NMR spectrum of 3-amino- $N$-phenyl-4,6-distyrylthieno[2,3-b]pyridine-2-carboxamide (6). In DMSO- $d_{6}$ 


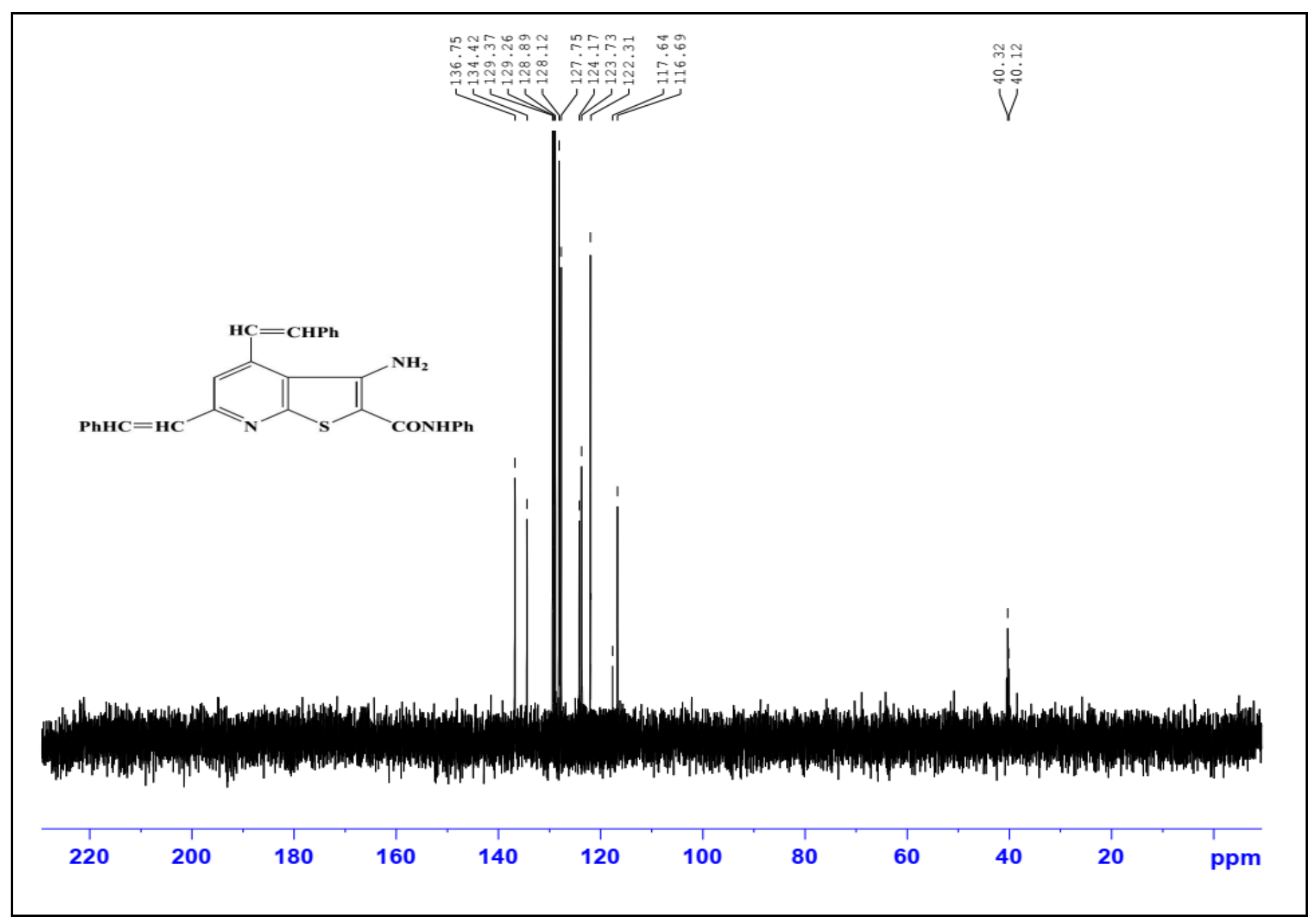

Figure S12: DEPT 135 spectrum of 3-amino-N-phenyl-4,6-distyrylthieno[2,3-b]pyridine-2-carboxamide (6). In DMSO- $d_{6}$ 


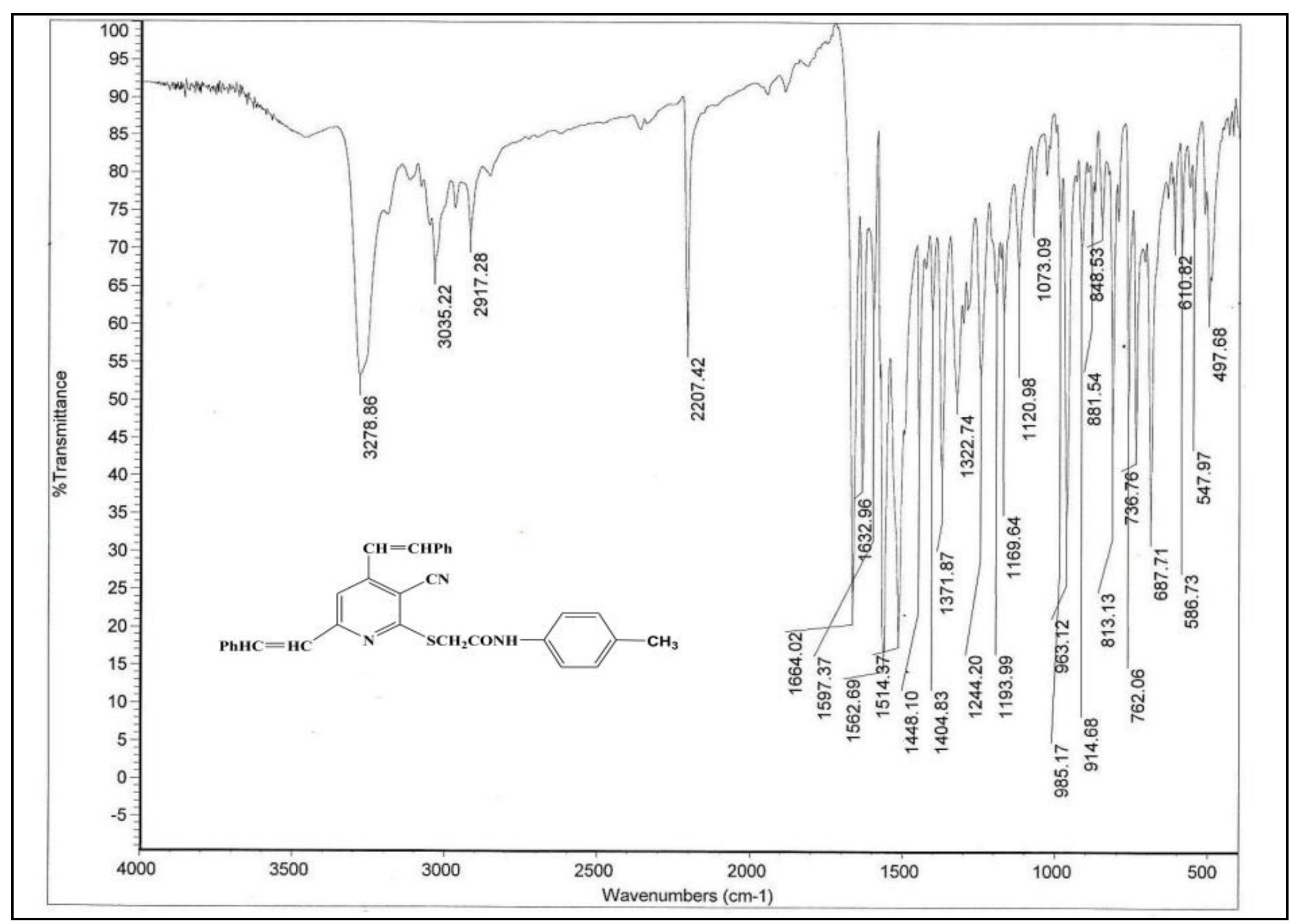

Figure S13: IR spectrum of 2-((3-cyano-4,6-distyrylpyridin-2-yl)thio)-N-(p-tolyl)acetamide (7). 


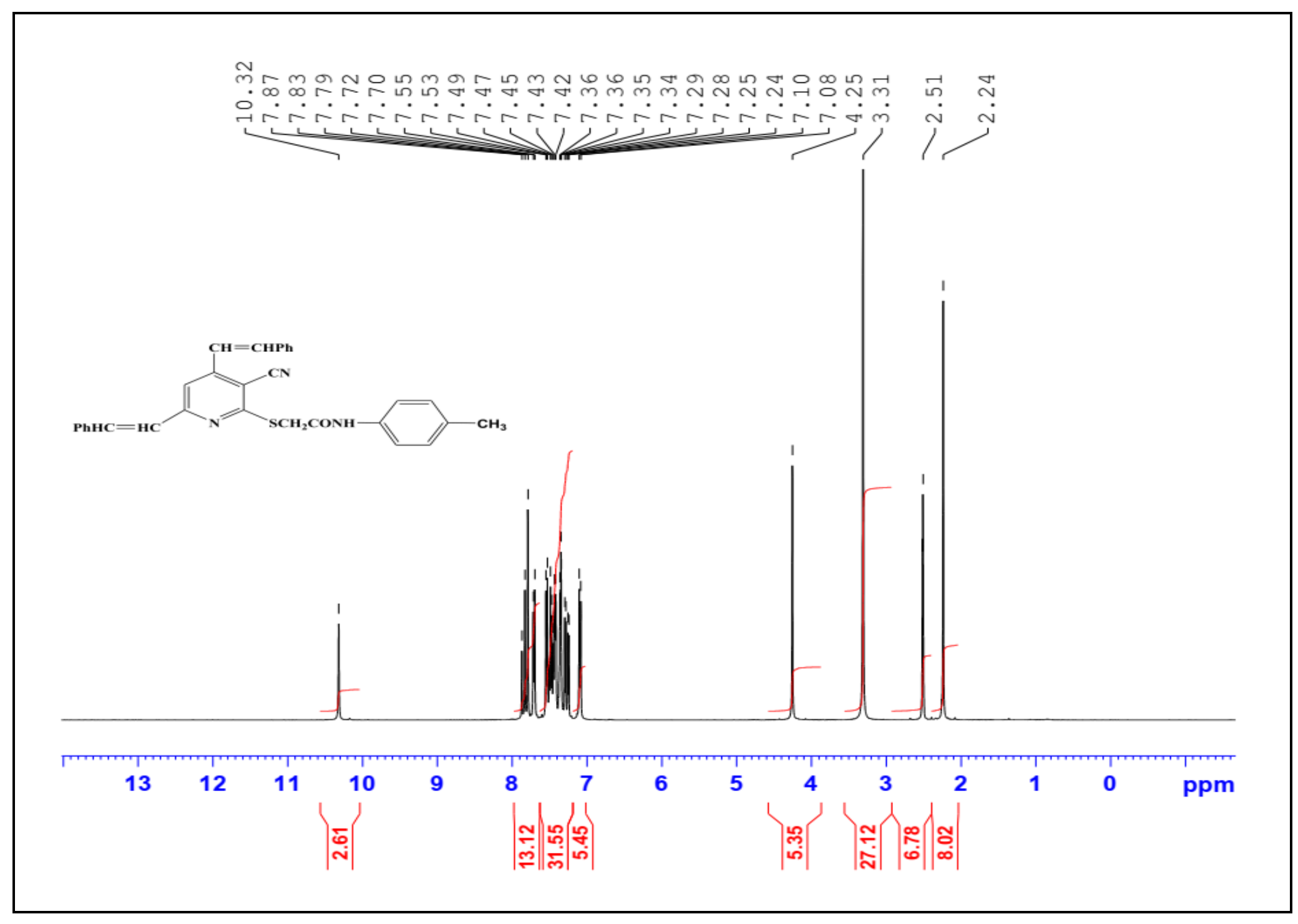

Figure S14: ${ }^{1} \mathrm{H}$ NMR spectrum of 2-((3-cyano-4,6-distyrylpyridin-2-yl)thio)- $N$-(p-tolyl)acetamide (7). In DMSO- $d_{6}$ 


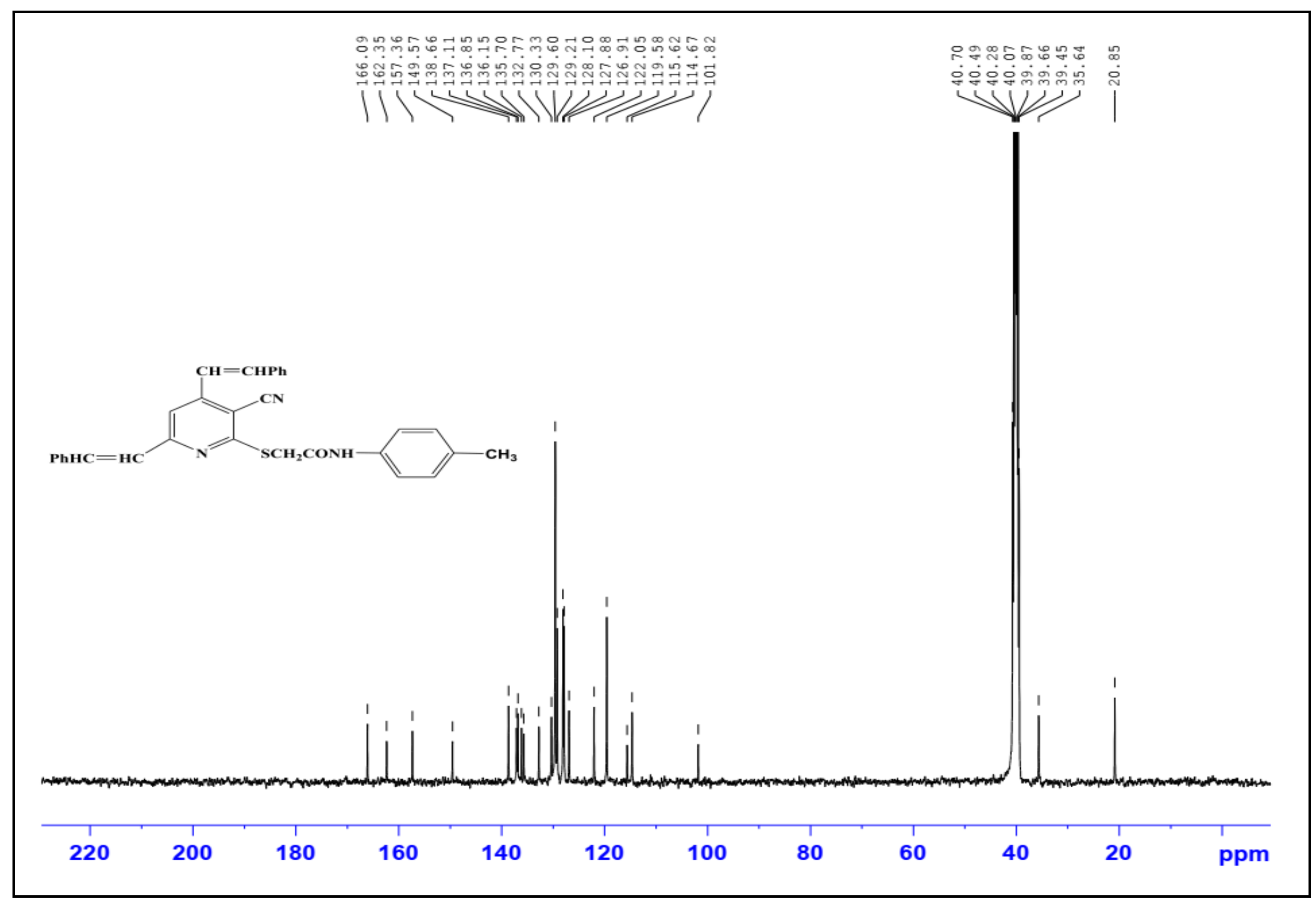

Figure S15: ${ }^{13} \mathrm{C}$ NMR spectrum of 2-((3-cyano-4,6-distyrylpyridin-2-yl)thio)-N-(p-tolyl)acetamide (7). In DMSO- $d_{6}$ 


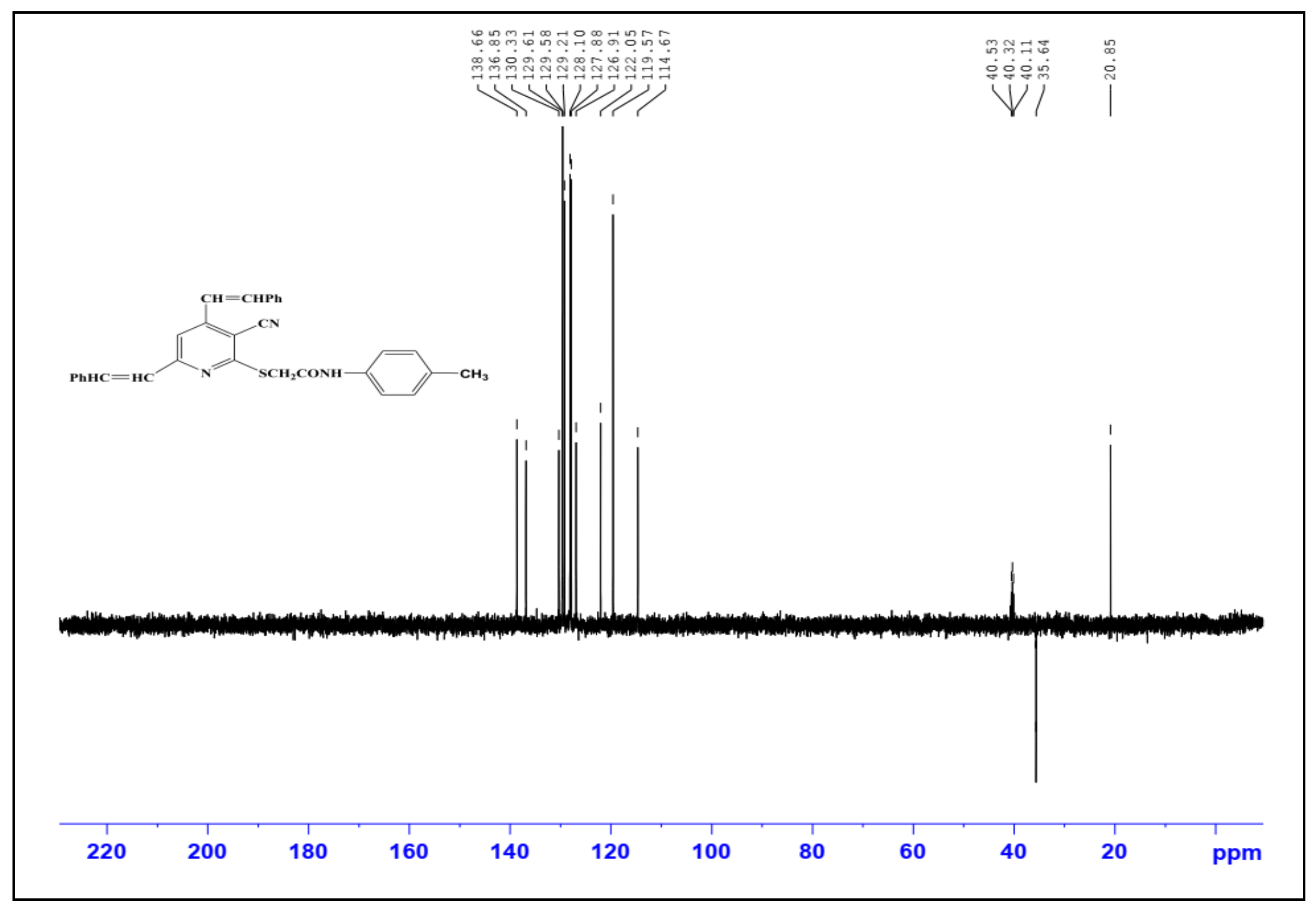

Figure S16: DEPT 135 spectrum of 2-((3-cyano-4,6-distyrylpyridin-2-yl)thio)- $N$-(p-tolyl)acetamide (7). In DMSO- $d_{6}$ 


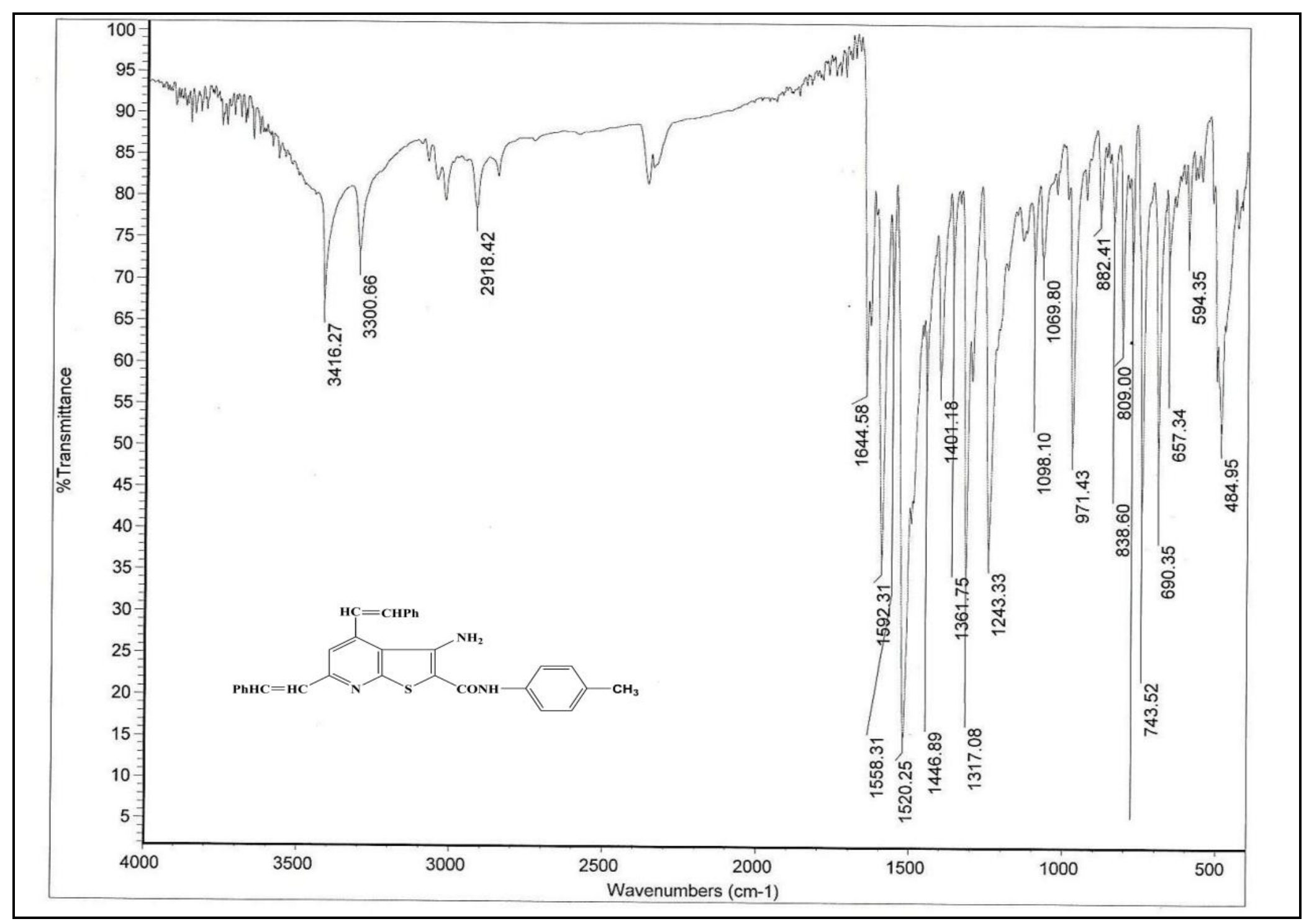

Figure S17: IR spectrum of 3-amino-4,6-distyryl- $\mathrm{N}$-(p-tolyl)thieno[2,3-b]pyridine-2-carboxamide (8). 


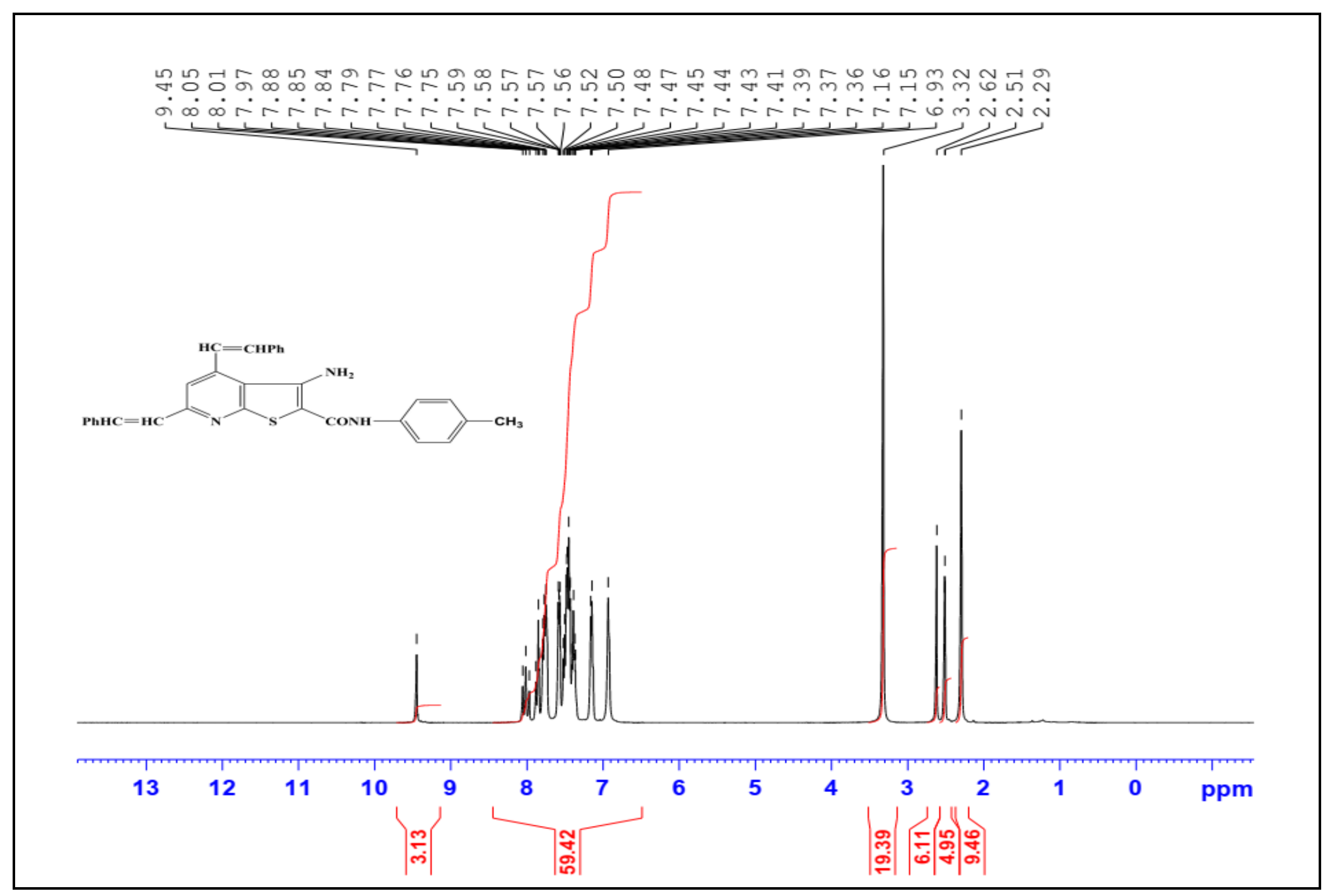

Figure S18: ${ }^{1} \mathrm{H}$ NMR spectrum of 3-amino-4,6-distyryl- $N$-(p-tolyl)thieno[2,3-b]pyridine-2-carboxamide (8). In DMSO- $d_{6}$ 


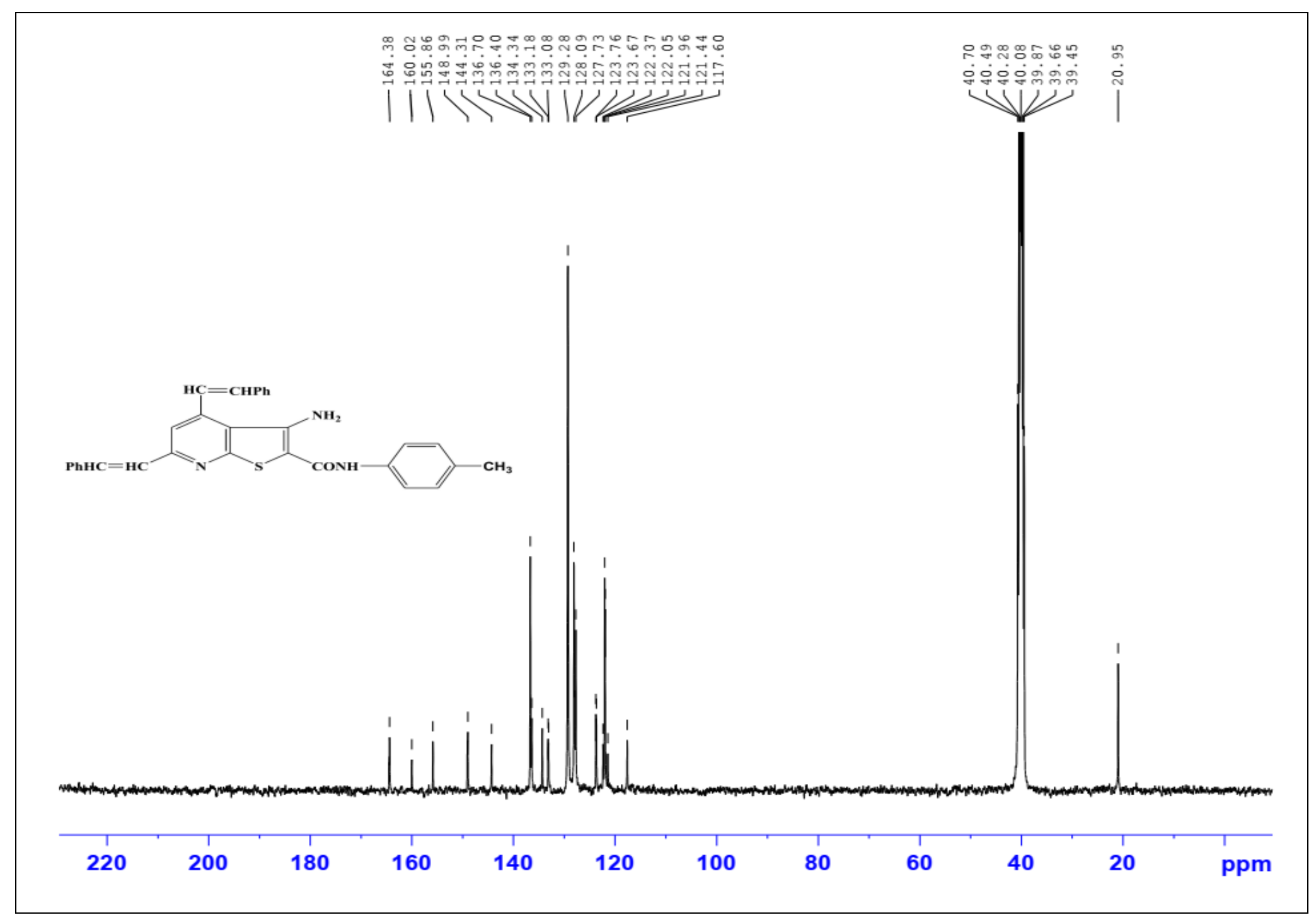

Figure S19: ${ }^{13} \mathrm{C}$ NMR spectrum of 3-amino-4,6-distyryl- $N$-(p-tolyl)thieno[2,3-b]pyridine-2-carboxamide $(\mathbf{8})$. In DMSO- $d_{6}$ 


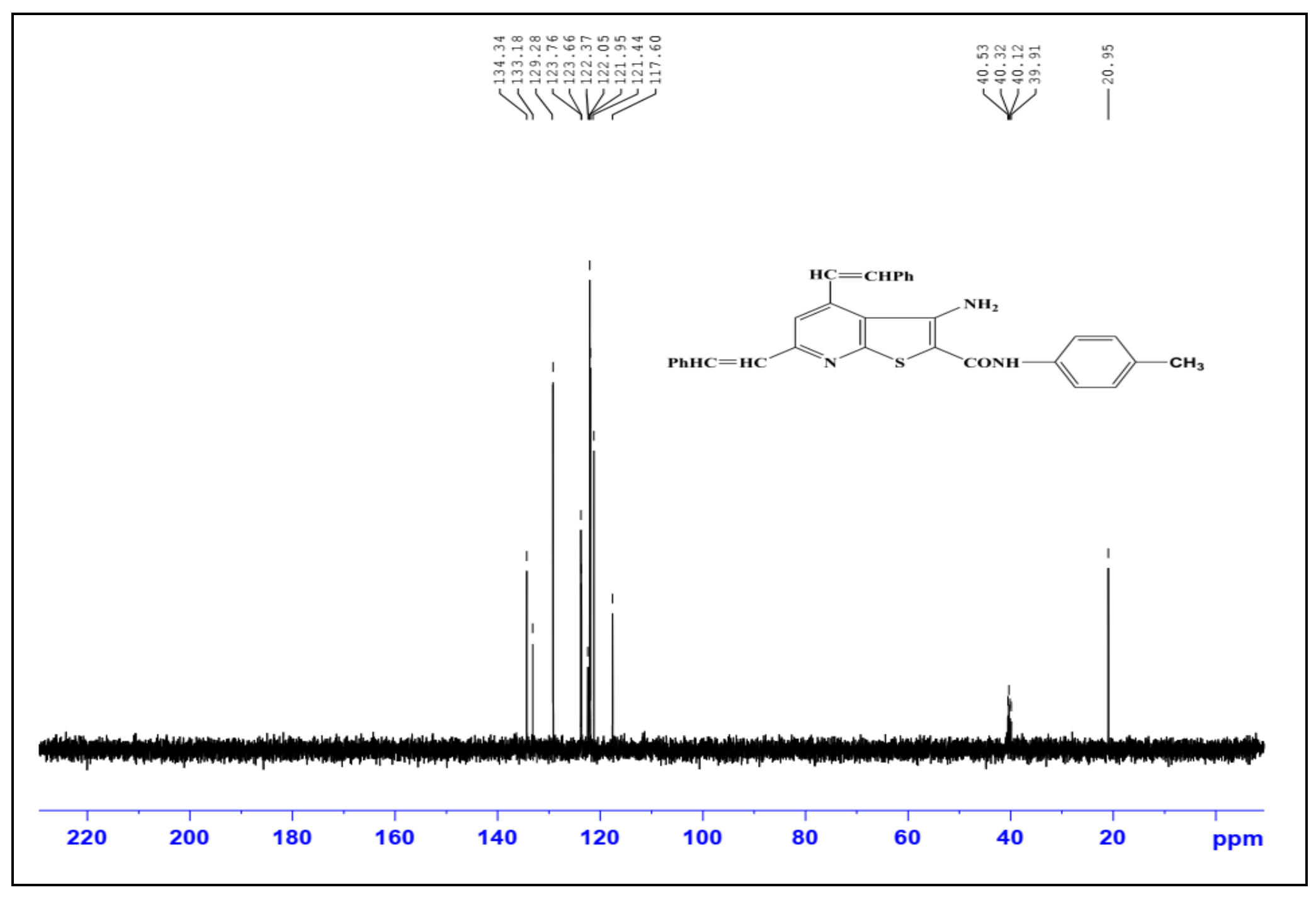

Figure S20: DEPT 135 spectrum of 3-amino-4,6-distyryl-N-(p-tolyl)thieno[2,3-b]pyridine-2-carboxamide (8). In DMSO- $d_{6}$ 


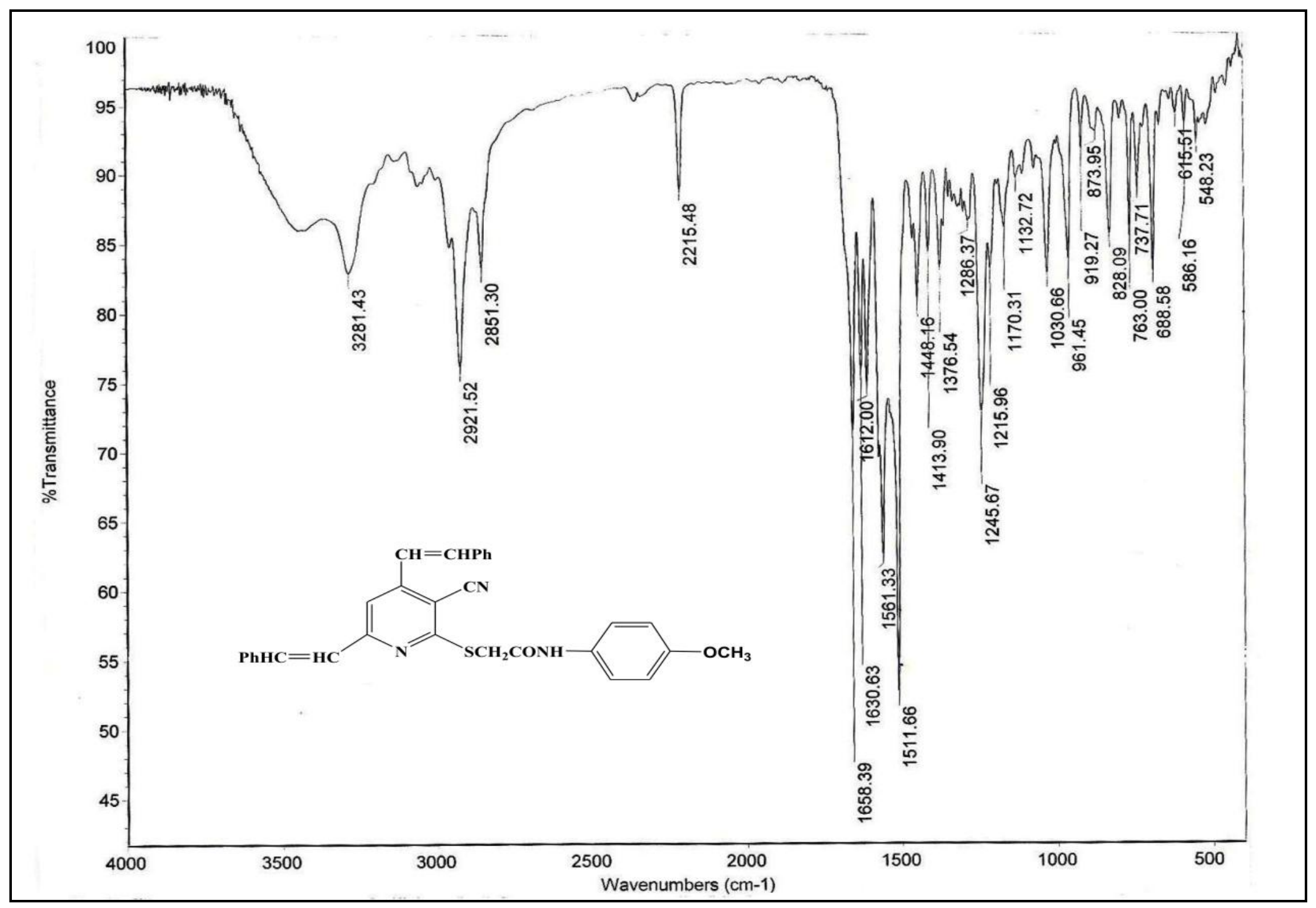

Figure S21: IR spectrum of 2-((3-cyano-4,6-distyrylpyridin-2-yl)thio)-N-(4-methoxyphenyl)acetamide (9). 


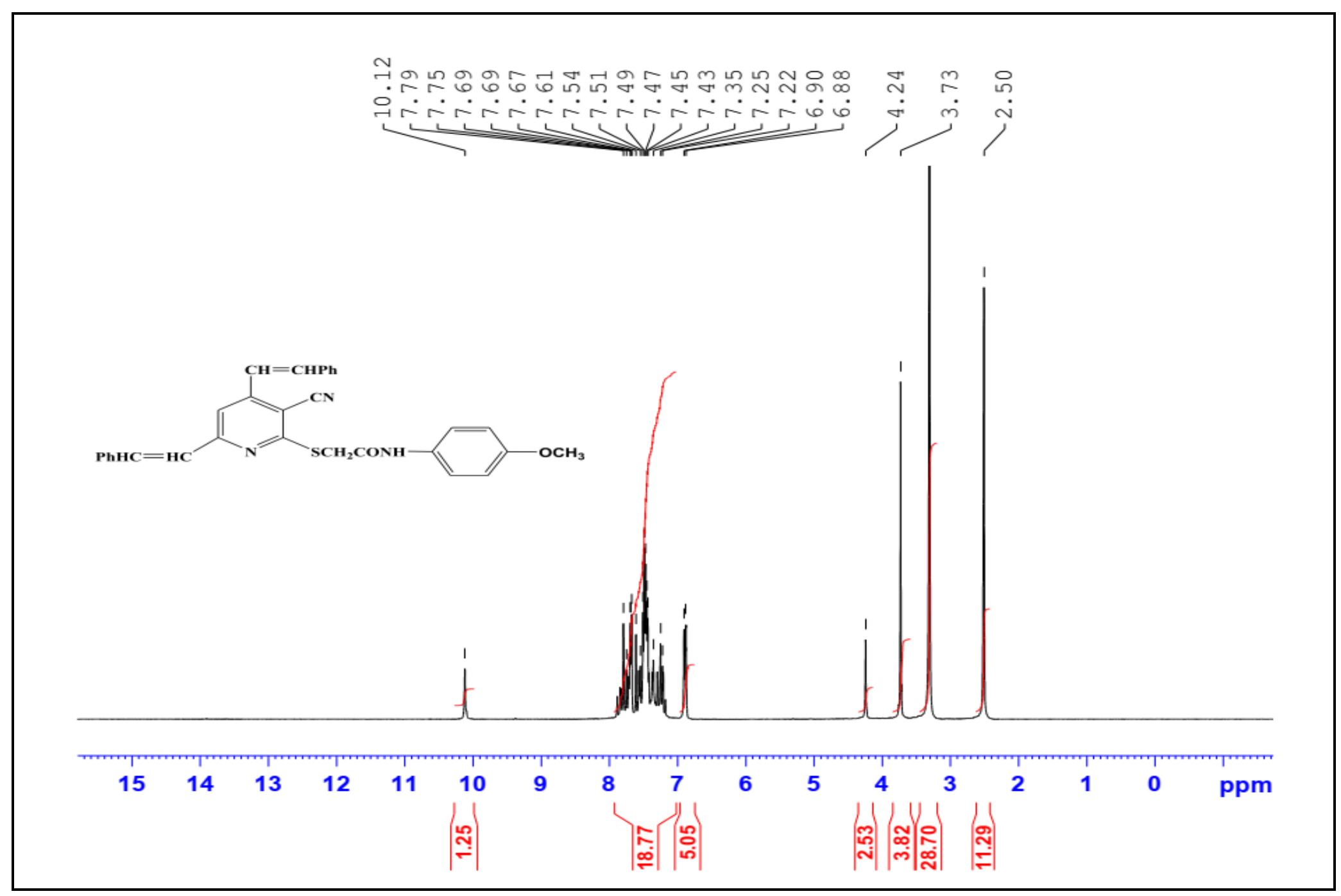

Figure S22: ${ }^{1} \mathrm{H}$ NMR spectrum of 2-((3-cyano-4,6-distyrylpyridin-2-yl)thio)-N-(4-methoxyphenyl)acetamide (9). In DMSO- $d_{6}$ 


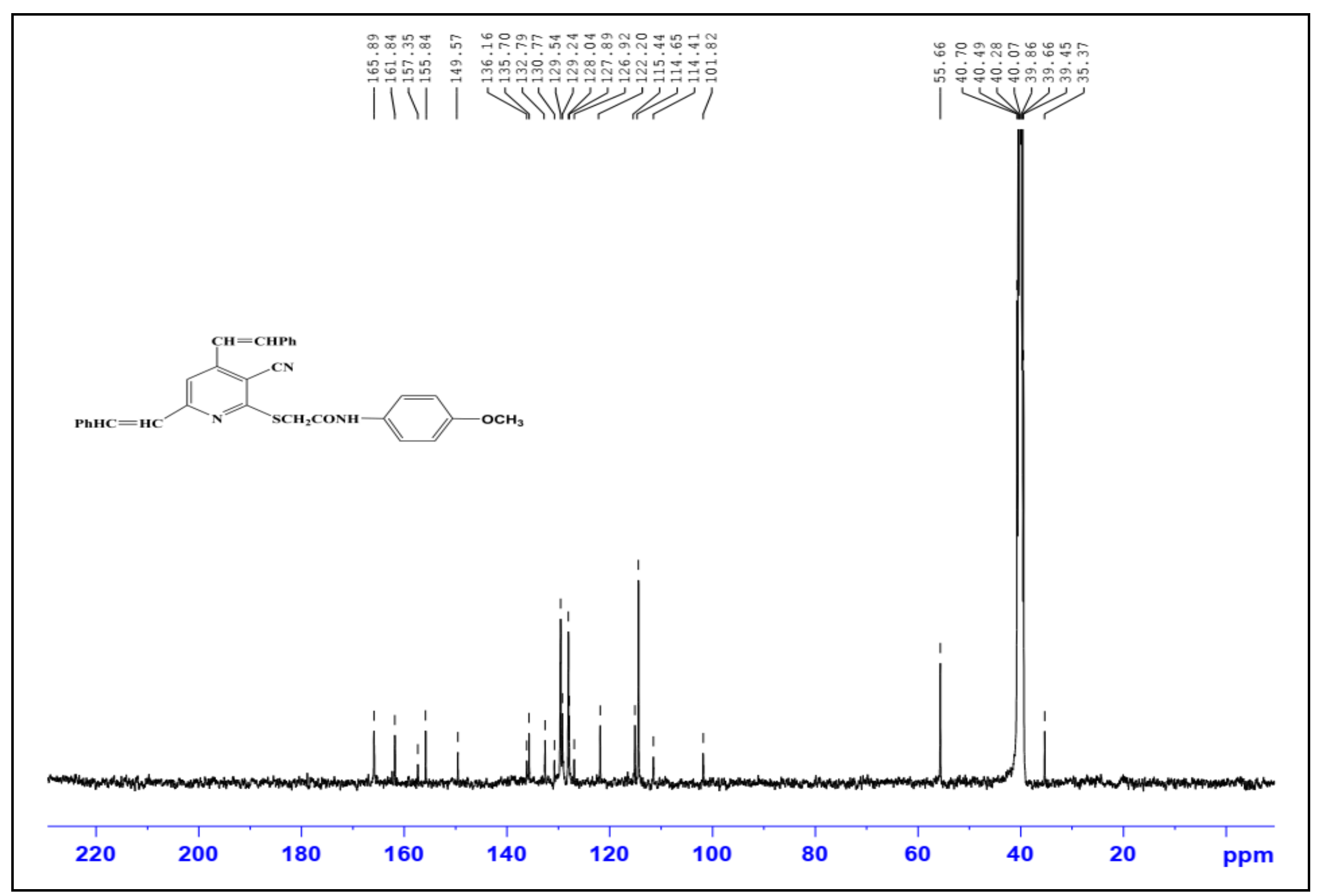

Figure S23: ${ }^{13} \mathrm{C}$ NMR spectrum of 2-((3-cyano-4,6-distyrylpyridin-2-yl)thio)-N-(4-methoxyphenyl)acetamide (9). In DMSO-d $d_{6}$ 


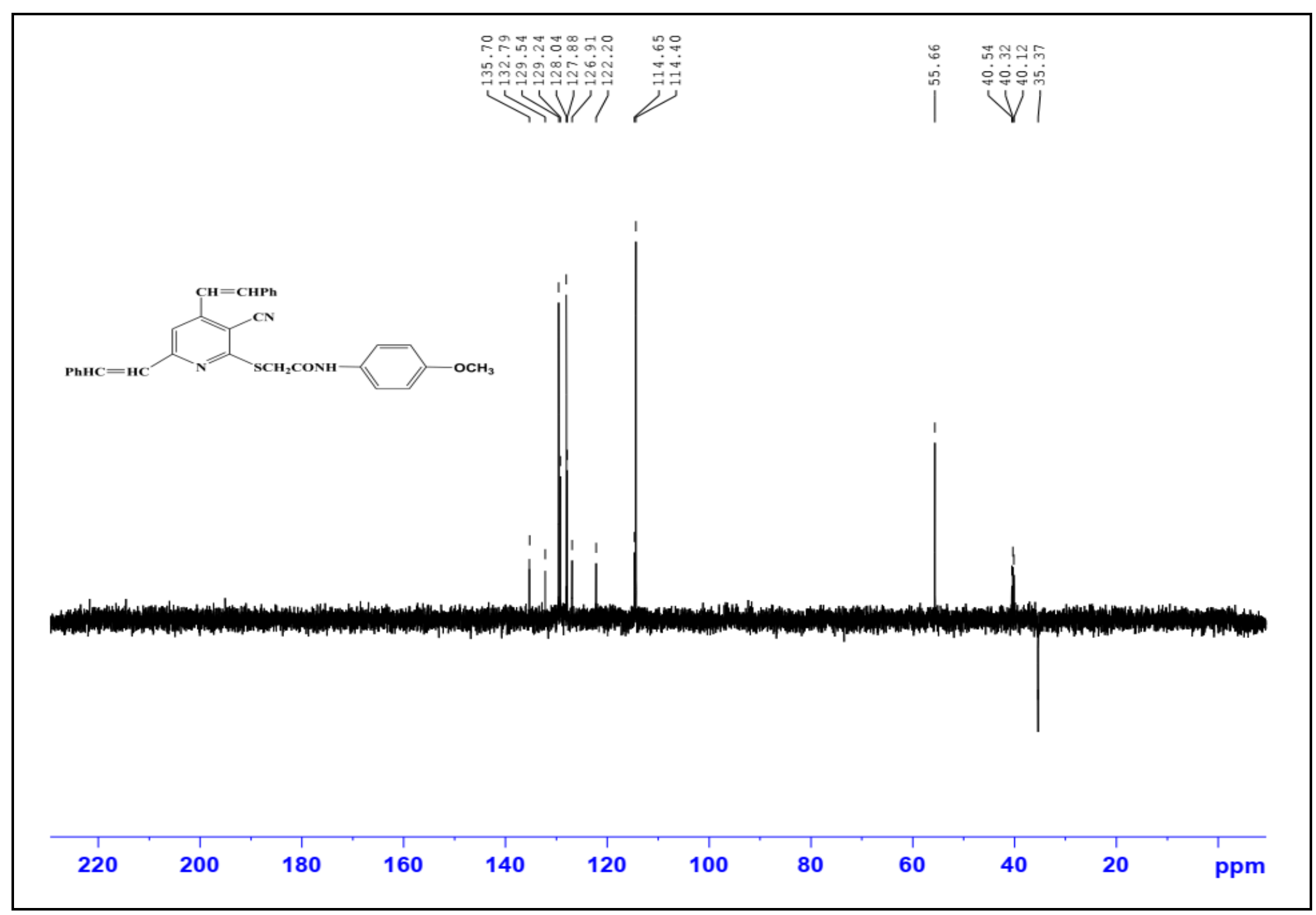

Figure S24: DEPT 135 spectrum of 2-((3-cyano-4,6-distyrylpyridin-2-yl)thio)-N-(4-methoxyphenyl)acetamide (9). In DMSO-d 6 


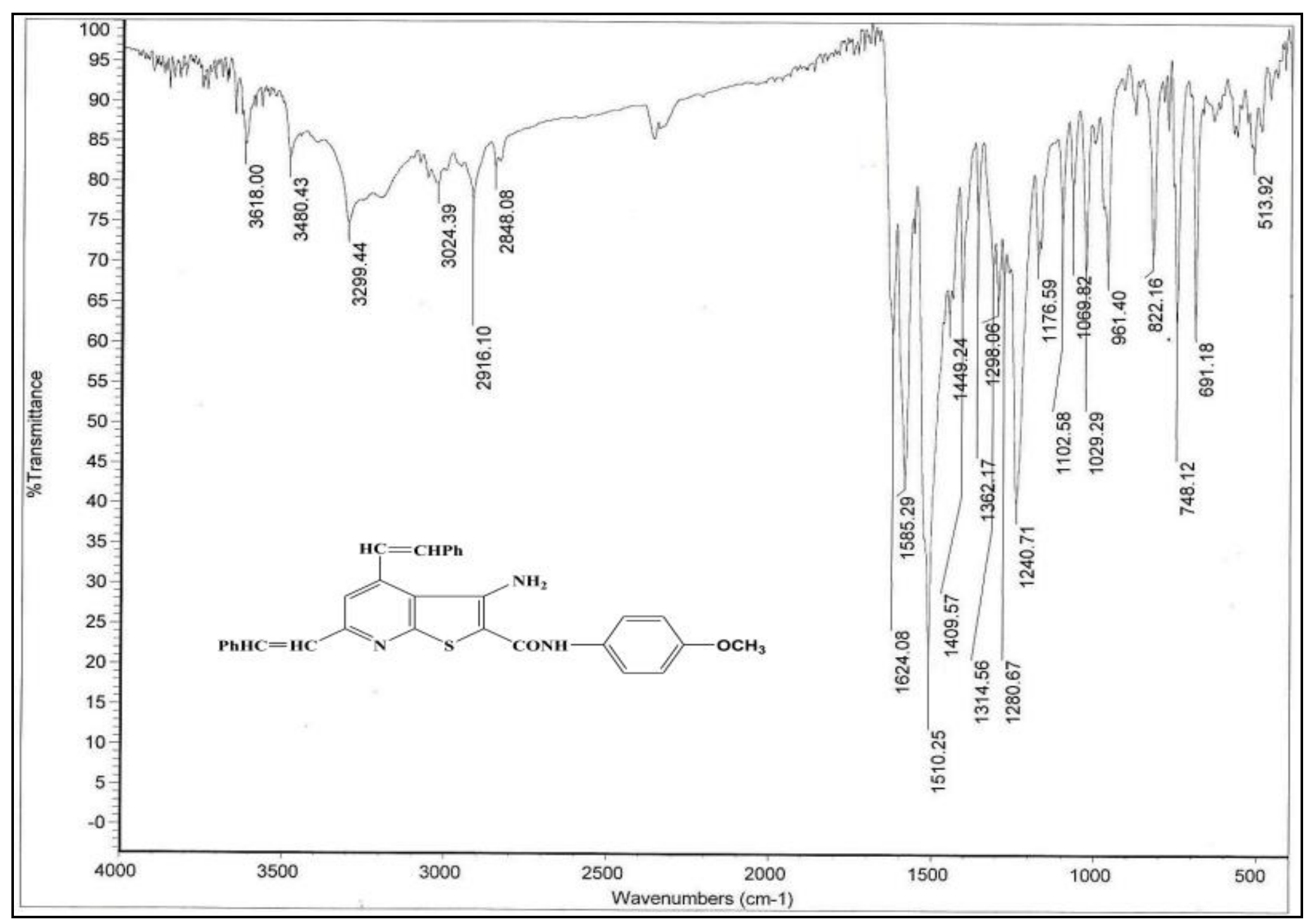

Figure S25: IR spectrum of 3-amino- $N$-(4-methoxyphenyl)-4,6-distyrylthieno[2,3-b]pyridine-2-carboxamide (10). 


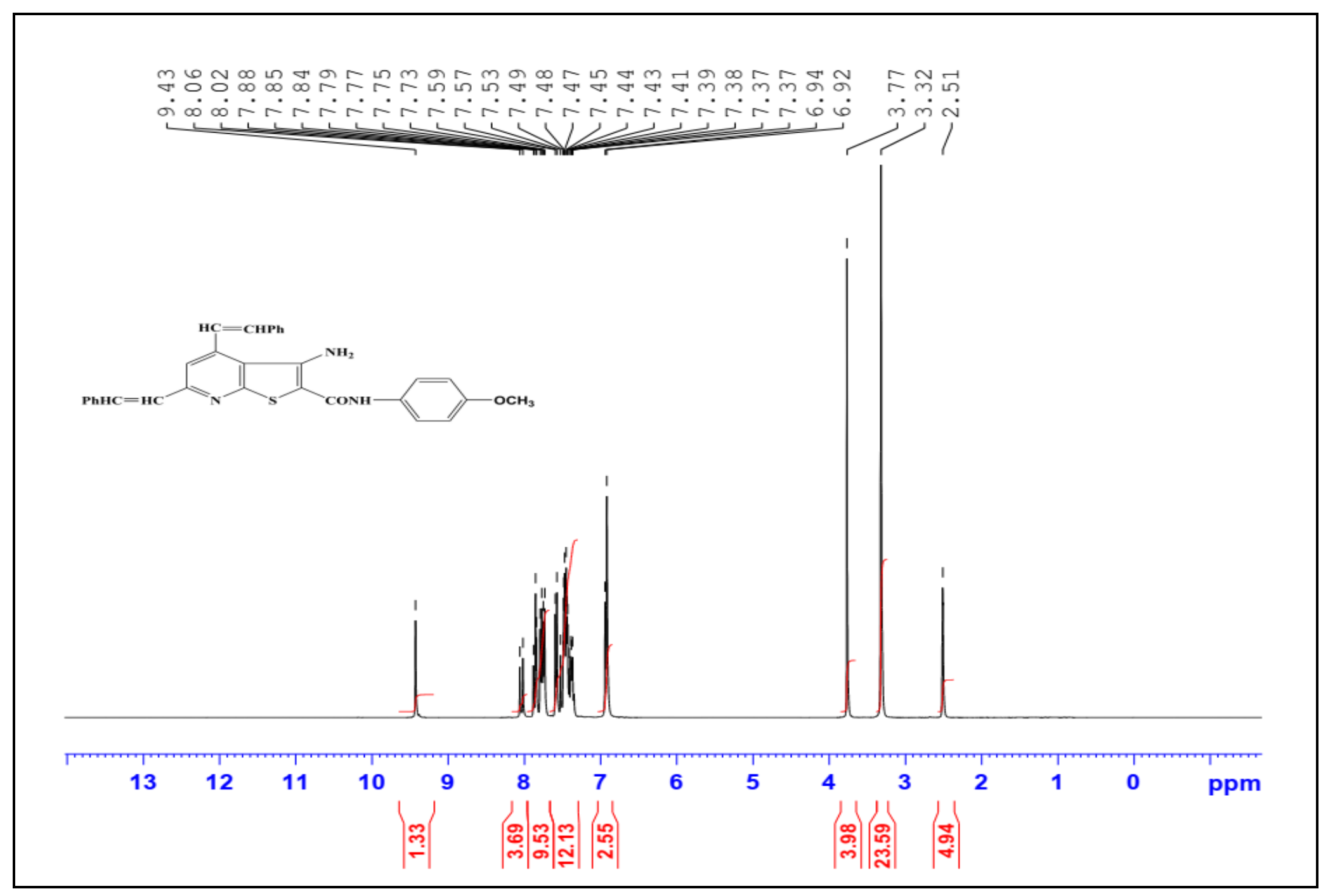

Figure S26: ${ }^{1} \mathrm{H}$ NMR spectrum of 3-amino-N-(4-methoxyphenyl)-4,6-distyrylthieno[2,3-b]pyridine-2-carboxamide (10). In DMSO- $d_{6}$ 


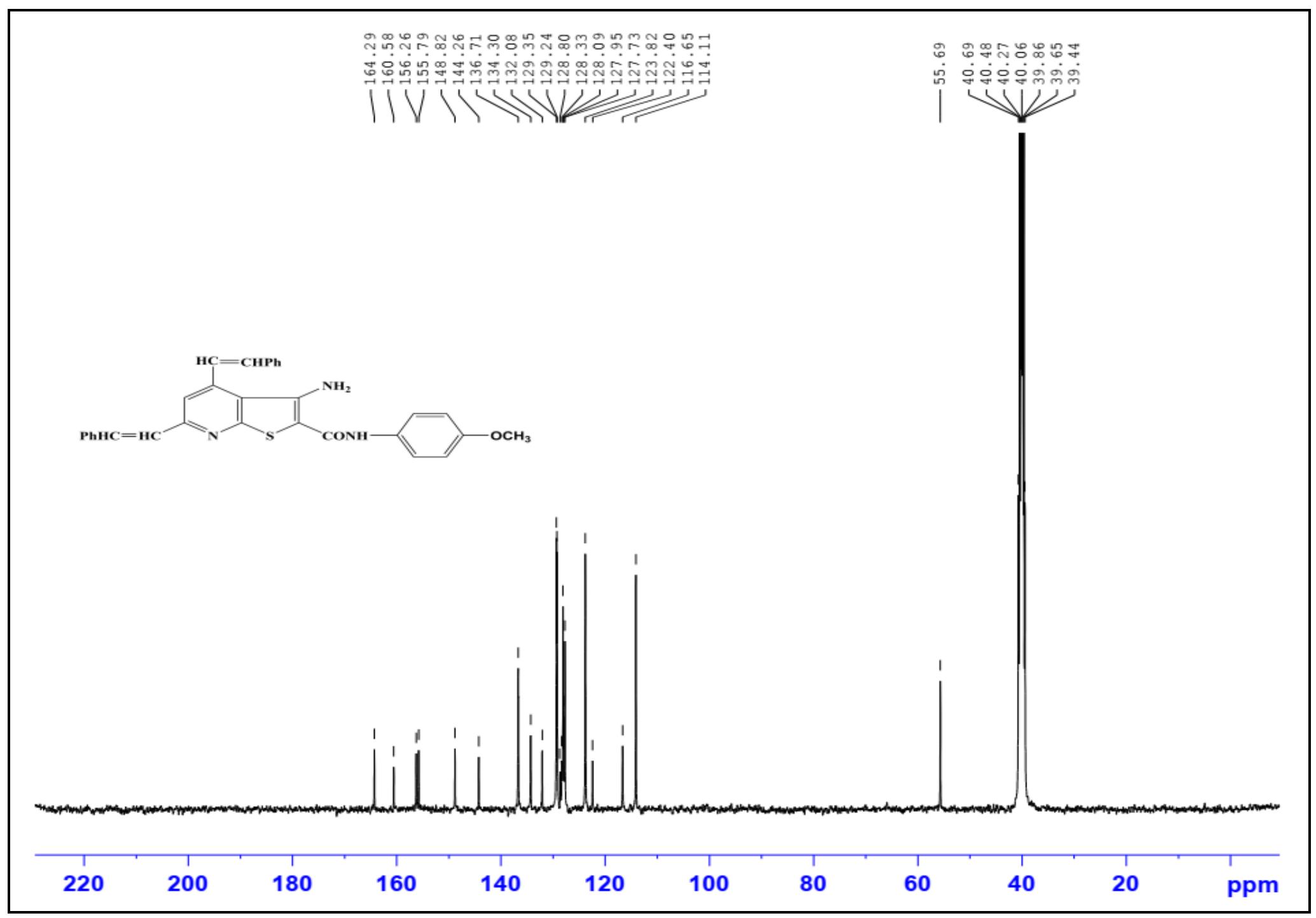

Figure S27: ${ }^{13} \mathrm{C}$ NMR spectrum of 3-amino-N-(4-methoxyphenyl)-4,6-distyrylthieno[2,3-b]pyridine-2-carboxamide (10). In DMSO-d 6

S28 


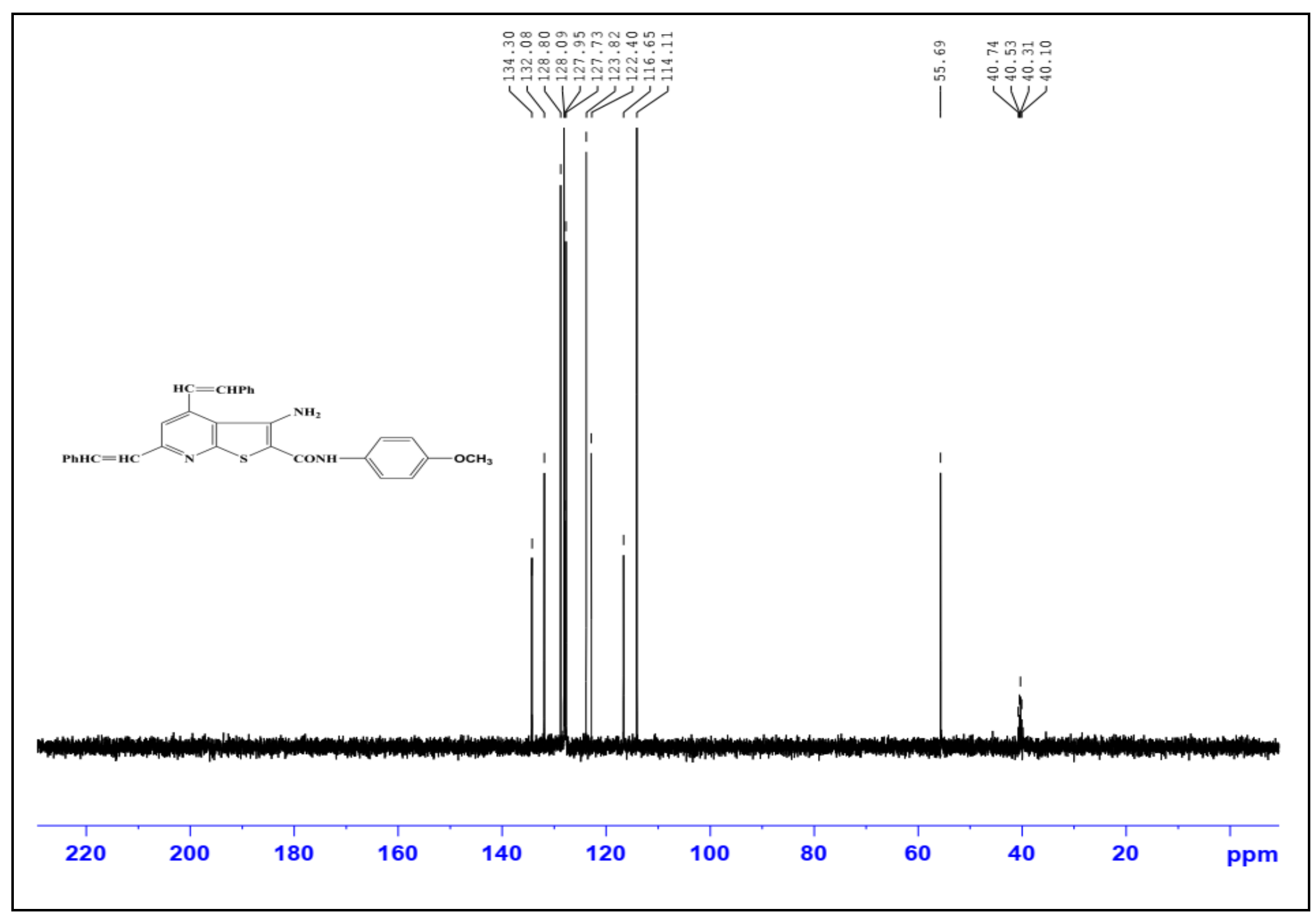

Figure S28: DEPT 135 spectrum of 3-amino-N-(4-methoxyphenyl)-4,6-distyrylthieno[2,3-b]pyridine-2-carboxamide (10). In DMSO-d 6 